\title{
Linear-time-varying feedforward control for position-dependent flexible structures
}

\author{
Fatemeh Sharifi $^{1}$, Hans Zwart ${ }^{2}$, Marcel Heertjes ${ }^{3}$, Nikolaos Kontaras ${ }^{4}$
}

\begin{abstract}
High performance motion systems in the semiconductor industry can achieve higher tracking accuracy when the structural dynamics of the system are taken into account. This work proposes an LTV feedforward control scheme which is based on the approximation of the system dynamics at low frequencies. The plant approximation contains both the low frequency contribution and resonance dynamics of the dominant modes. As an example of a flexible structure, the damped Euler-Bernoulli beam is investigated with considering the stable inversion of the approximated plant for feedforward control design. The results show a remarkable increase in positioning accuracy of the system being the consequence of the presence of feedforward control forces even when the reference setpoint accelerations equal zero.
\end{abstract}

\section{INTRODUCTION}

Wafer scanners in the semiconductor industry are the key tool in the manufacturing of integrated circuits (ICs). The demand for higher throughput of these scanners requires more aggressive motion profiles and larger forces. Consequently, a shift toward more lightweight designs rather than the use of extra amplifiers and actuators to support these forces, has been observed. The resulting decrease in mass however allows for an increased expression of internal flexibilities while scanning, which is experienced as position-dependent (time-varying) dynamics [2].

During the production of chips, a circular silicon wafer is positioned atop the wafer stage. This wafer is subjected under the exposure of ultraviolet (UV) light containing the blueprint of the ICs to be processed. After exposure, the specific pattern is etched away and one layer of integrated circuits is produced. This procedure is repeated several times to create ICs. The schematic of the wafer stage during exposure is illustrated in Fig. 1. Note that the position as an output of the system has to be translated from the edges

*This research has received funding from the European Unions Horizon 2020 research and innovation programme under the Marie SklodowskaCurie grant agreement No. 765579

${ }^{1}$ Fatemeh Sharifi is with the Faculty of Electrical Engineering, Mathematics and Computer Science, University of Twente, 7500 AE Enschede, The Netherlands s.f.sharifi@utwente.nl

${ }^{2}$ Hans Zwart is with the Faculty of Electrical Engineering, Mathematics and Computer Science, University of Twente, 7500 AE Enschede, The Netherlands, and Dynamics and Control group, Eindhoven University of Technology, 5612 AZ Eindhoven, The Netherlands h.j.zwarteutwente.nl, h.j.zwartetue.nl

${ }^{3}$ Marcel Heertjes is with ASML, Mechatronic System Development, Veldhoven, The Netherlands, and Dynamics and Control group, Eindhoven University of Technology, 5612 AZ Eindhoven, The Netherlands marcel.heertjes@asml.com, m.f.heertjes@tue.nl

${ }^{4}$ Nikolaos Kontaras is with Dynamics and Control group, Eindhoven University of Technology, 5612 AZ Eindhoven, The Netherlands nkontaras@brainpeace.nl of the stage, where the sensors are located for measuring, toward the point-of-interest (POI) which is located under the UV lens. In order to conduct the translation from the

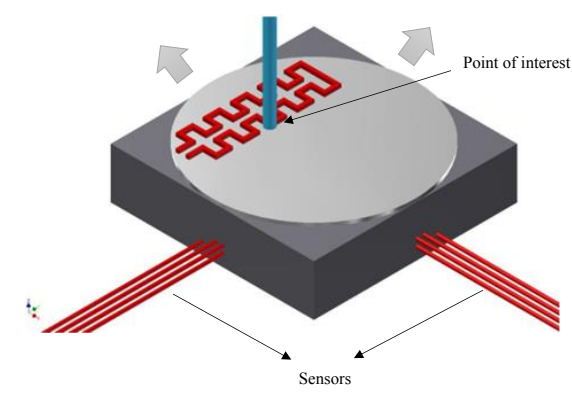

Fig. 1: Wafer stage during exposure. The sensors measure the output at a different location from exposure, which requires a dynamic transformation to obtain the latter point-of-interest.

measured location toward the POI, a rigid body (RB) mode decoupling strategy is conducted. This in combination with traditional acceleration feedforward control cannot account for the effect of the non-rigid body (NRB) modes of the flexible system [3]. In order to improve tracking performance at low frequencies for flexible structures, snap feedforward control is usually applied [1]. Snap feedforward control adds a jerk derivative to the traditional acceleration feedforward control structure. The drawback of this feedforward control approach is that it only deals with the quasi-static frequency contribution of NRB modes and does not sufficiently address the resonant behaviour. Furthermore, being linear-timeinvariant (LTI) it cannot deal with time-varying dynamics as occurring in the wafer scanning example of Fig. 1. In [4] adaptive feedforward control is investigated with the aim of reducing the peak position error by creating positionindependent behaviour. In other words, the adaptation adjusts the mass-parameter (the mass of the system does not change) in order to deal with flexibilities.

Other approaches that consider the plant as linear-timevarying (LTV) or Linear parameter varying (LPV) are for example given by [5], where a method is proposed to design a gain-scheduled inverse of an LPV system. In this method, an infinite number of Linear Matrix Inequalities (LMIs) has to be solved. This is solved in [6], while the solution is again depending on LMIs, and this causes that the higherorder systems might not yield tractable solutions. In fact, as 
a disadvantage, this method requires a sufficiently accurate parametric model, which in practice is often hard to obtain. Two recent approaches toward feedforward control design for LTV and linear parameter varying (LPV) systems are studied in [7] and [8]. [7] studies the exact plant inversion of the LTV system with a parametric model. It derives conditions in order to achieve an unique feedforward control signal. [8] investigates the optimal feedforward control design for LTV/LPV systems. The feedforward control signal is constructed by a linear combination of basis functions which have optimal coefficients computed by a quadratic optimization process. Here Gaussian radial functions are chosen as the signal bases since they are compatible with arbitrary input signals. Experimental validation of the above two feedforward control technique is studied in [9]. The methods are performed on a single-input-single-output (SISO) rotational two-mass system. The measured results demonstrate improvement in tracking performance in comparison to acceleration feedforward control.

Different from the previous approaches, in [11] compliance compensation is discussed as feedforward control technique for time-varying motion systems. This method adds the quasi-static behaviour of flexible structures as a correction block to the control structure. Later, in [12], the former compliance feedforward control is tuned by a low-pass filter to an LTV system. It retains the control objectives of snap feedforward control for an LTV system. Similar to [11], it mostly accounts for the compliant part of the positiondependent structure, since it only considers a low frequency approximation of the plant. In a more recent extension [13], LTV feedforward control addresses one resonance of the flexible structure as well as the low frequency contribution of the NRB modes as a whole. It has been shown that the performance of this system is much improved. The method is validated for a rotational two-mass-spring-damper system. Following the previous works, specially [12], [13], the main contribution of this work is to design a feedforward controller for an LTV system in which the stable plant inversion is based on an LTV fourth-order model. The model considers the quasi-static deformation of the plant besides the dominant resonant dynamics of the system. The feedforward controller improves the accuracy in tracking the desired output. Furthermore, the model is validated for the damped Euler-Bernoulli beam, which is different from [13].

The outline of this work is as follows, in section II the mathematical representation of the time-varying flexible motion system is introduced. Section III presents the overall challenges in the control design of flexible systems. The typical model-based feedforward control and its restrictions for an LTV system are discussed. Next, the new LTV feedforward control is discussed in order to cover the limitations of LTV model-based snap feedforward control. Section IV investigates criteria of feasibility for the plant inversion. Section V presents the Euler-Bernoulli beam which serves as a simplified case of a flexible LTV system. The simulated model validates the proposed feedforward control scheme. Some concluding remarks are given in section VI.

\section{SYSTEM DESCRIPTION}

The wafer stage is a double-integrator-based flexible system that includes, in a simplified representation, one RB mode and an infinite number of NRB modes. The transfer of these class of systems in time-domain representation is given by

$$
G(p)=\frac{X(p)}{F(p)}=\frac{1}{m p^{2}}+G_{N R B s}(p),
$$

where $G_{N R B s}$ is the transfer of all NRB modes. It should be noted that the time-varying feature of the system modifies the mathematical description of the system, the operator $p$ is introduced as the time differentiation operator rather than presenting a frequency-domain description based on the Laplace symbol $s$, see also Section 2.3 of [14]. This operator $p$ works on the complete time function, see e.g. the last line of (2).

Since the system is only performing in the low frequency range, the plant can be approximated by

$$
\begin{array}{r}
\tilde{G}(p, t)=\frac{Y(p)}{U(p)}=\frac{1}{m p^{2}}+C(t) \Leftrightarrow \\
y(t)=\frac{1}{m} \iint u(\tau) d \tau+C(t) u(t) \Leftrightarrow \\
m \ddot{y}(t)=u(t)+m \frac{d^{2}(C(t)) u(t)}{d t^{2}},
\end{array}
$$

where $C(t)$ is the total compliance of the system as a function of time $t$. It is representative for the quasi-static behaviour of the entire NRB dynamics until the occurrence of the first resonance. Hence, the need to incorporate many NRB modes at low frequencies can be relaxed by only taking into account the compliance part of the system.

\section{CONTROL PROBLEM}

Regarding the wafer stage, there are two main problems that need to be considered for feedforward control design. Firstly, the structural dynamics and resonance of each NRB mode, which due to the lightweight structure, are substantial and should be taken into account. Secondly, the LTV nature of the flexible system needs to be considered. In other words, as depicted in Fig. 1, the sensors for measuring the position are located at the edges of the stage, while the operation takes place on the wafer in the area around the POI. Thus, the measured output is not representing the ongoing output of the system. Therefore, the feedforward controller is required to take into account both the time-varying feature of the POI as well as the flexible dynamics of the system.

\section{A. Model-based feedforward controller}

The main aim of the control action in our position-tracking problem is to obtain the desired output which is a pre-known function. Perfect tracking of setpoint trajectories can often be fairly achieved by the use of feedforward control. In other words, the model-based feedforward control design targets at diminishing the error between the real measured output and the desired output. 
Fig. 2 shows the typical control scheme of the system. The model-based feedforward controller (denoted by $F F$ ), which approximates the inverse of the system dynamics $G$, can help to achieve perfect tracking, provided that the solution for the inverse problem exists. For example, due to the presence of non-minimum phase (NMP) zeros, the inverse plant may become unstable. Hence, the use of an inverse model can often only be obtained in some approximate sense. A straightforward model-based feedforward controller

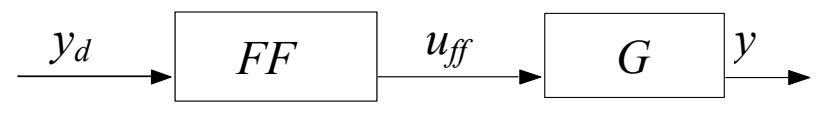

Fig. 2: Model-based feedforward control scheme

is the acceleration/mass feedforward control, which is the inversion of only the rigid body mode of the system. It cannot compensate for the behaviour of the flexibility of the structural dynamics since it ignores the NRB modes. In other words this model-based feedforward controller employs only the acceleration term of the reference setpoint as a feedforward controller in a typical control scheme, i.e., the $F F$ block in Fig. 2. Obviously, the mentioned problems in model-based feedforward control can occur with this conventional controller which makes it often insufficient for an LTV system to obtain sufficiently high accuracy.

The new proposed feedforward controller tries to improve the accuracy for both LTI/LTV systems, with a modified (and invertible) approximated plant.

\section{B. LTV snap feedforward control}

The position-dependent compliance feedforward control scheme is visualized in Fig. 3. This feedforward controller is an LTV extension of snap feedforward control [1]. Note that the latter cannot compensate for LTV systems [12].

The POI $r_{p}=r_{p}(t)$, is varying with respect to time $t$ and affects the feedforward controller design. $G\left(r_{p}\right)$ is a flexible system with position-dependent parameters. $F F_{c c}\left(r_{p}\right)$ is the new model-based feedforward controller which is based on the inversion of the approximated plant $\tilde{G}(p, t)$ at low frequencies, see (2). It can be obtained symbolically by the following:

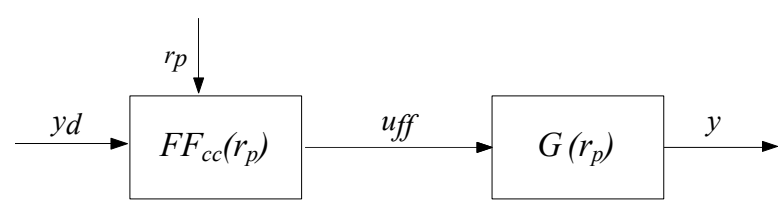

Fig. 3: LTV snap feedforward control

$$
F F_{c c}(p, t)=\tilde{G}^{-1}(p, t)=\frac{m p^{2}}{1+m p^{2} C(t)} .
$$

For the LTI case $(C(t)=C),(3)$ renders the snap feedforward control which is the fourth-order Taylor expansion of the inversion of the approximated plant $\tilde{G}^{-1}(p)$, namely,

$$
F F_{c c}(p)=\frac{m p^{2}}{1+m p^{2} C}=m p^{2}-m^{2} p^{4} C+\mathscr{O}\left(p^{6}\right),
$$

or

$$
F F_{c c}(p)=k_{f a} p^{2}+k_{f s} p^{4},
$$

with $k_{f a}=m$ and $k_{f s}=-m^{2} C$. Since the wafer stage problem is an LTV system, the new LTV feedforward control is proposed. From (3), $u_{f f}=F F_{c c}(p, t) y_{d}(t)$ gives

$$
\begin{aligned}
& u_{f f}(t)+m \frac{d^{2}}{d t^{2}}\left(C(t) u_{f f}(t)\right)=m \frac{d^{2}}{d t^{2}} y_{d}(t) \Leftrightarrow \\
& \ddot{u}_{f f}(t)=-\frac{2 \dot{C}(t)}{C(t)} \dot{u}_{f f}(t)-\frac{1+m \ddot{C}(t)}{m C(t)} u_{f f}(t)+\frac{1}{C(t)} \ddot{y}_{d}(t) .
\end{aligned}
$$

For the LTV case, the stability of the feedforward controller (6) is more complicated and problematic, since no practical and sufficient condition is available. For instance, for a simple exponential compliance function, the calculated feedforward control input becomes extremely large and unbounded. In order to solve this problem, a new approximation of the plant at low frequencies is proposed in the next section which improves the stability of the feedforward control for both the LTI/LTV cases.

\section{LTV feedforward control design}

In this section, the plant will be approximated at low frequencies by a low pass filter. This filter not only restricts the input signal at low frequency content, but also adds a resonance frequency as an approximation of the dominant mode to the plant approximation. The control scheme is identical to Fig. 3. The approximated plant to be inverted is depicted in Fig. 4.

For the aim of feedforward control design, exact inversion

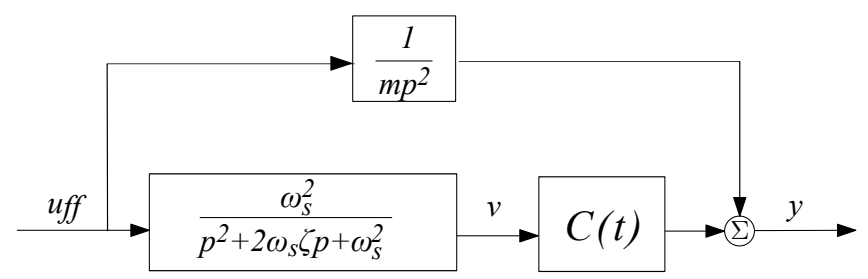

Fig. 4: Low-frequency approximation of plant $\tilde{G}_{\omega}$ for the model-based feedforward controller

of this approximated plant generates the feedforward control signal $u_{f f}$ as the output given a pre-defined output trajectory. This approximate model consists of two governing equations namely,

$$
\begin{array}{r}
\tilde{G}_{\omega}(p, t)=\frac{1}{m p^{2}}+C(t) \frac{\omega_{s}^{2}}{p^{2}+2 \omega_{s} \zeta p+\omega_{s}^{2}} \Leftrightarrow \\
m \ddot{y}(t)=u_{f f}(t)+m \frac{d^{2}}{d t^{2}}(C(t) v(t)),
\end{array}
$$

with

$$
\ddot{v}(t)+2 \omega_{s} \zeta \dot{v}(t)+\omega_{s}^{2} v(t)=\omega_{s}^{2} u_{f f}(t),
$$


where $\omega_{s}>0$ is the dominant resonance frequency and $\zeta>0$ is the damping coefficient.

From (7) and (8) we conclude that the relation between $v(t)$ and $y(t)$ is given by

$$
\omega_{s}^{2} m \ddot{y}(t)=\ddot{v}(t)+2 \omega_{s} \zeta \dot{v}(t)+\omega_{s}^{2} v(t)+\omega_{s}^{2} m \frac{d^{2}(C(t) v(t))}{d t^{2}} .
$$

Hence, if the desired output is given $\left(y(t)=y_{d}(t)\right)$, then we obtain

$$
\ddot{v}(t)=\kappa_{1}(t) \dot{v}(t)+\kappa_{2}(t) v(t)+\kappa_{3}(t) \ddot{y}_{d}(t)
$$

where

$$
\begin{gathered}
\kappa_{1}(t)=-\frac{2 \omega_{s}\left(\omega_{s} m \dot{C}(t)+1\right)}{\omega_{s}^{2} m C(t)+1}, \\
\kappa_{2}(t)=-\frac{\omega_{s}^{2}(m \ddot{C}(t)+1)}{\omega_{s}^{2} m C(t)+1}, \\
\kappa_{3}(t)=+\frac{\omega_{s}^{2} m}{\omega_{s}^{2} m C(t)+1} .
\end{gathered}
$$

The control input of the new model is given by, see (8)

$$
u_{f f}(t)=\underbrace{\frac{1}{\omega_{s}^{2}}}_{\mu_{1}} \ddot{v}(t)+\underbrace{\frac{2 \zeta}{\omega_{s}}}_{\mu_{2}} \dot{v}(t)+v(t) .
$$

In order to find the signals $v(t), \dot{v}(t)$, and $\ddot{v}(t)$, the above equations will be solved through numerical integration for a given $y_{d}(t)$. This will be done later in the simulation section. Knowing the parametric model as well as the relative degree of the system, the unique feedforward control signal will be assured [7]. Hence, the LTV feedforward controller $F F_{c c}$ can be written in state-space form as

$$
\begin{gathered}
\dot{x}(t)=\underbrace{\left[\begin{array}{cc}
0 & 1 \\
\kappa_{2}(t) & \kappa_{1}(t)
\end{array}\right]}_{A_{F F}(t)} x(t)+\underbrace{\left[\begin{array}{c}
0 \\
\kappa_{3}(t)
\end{array}\right]}_{B_{F F}(t)} \ddot{y}_{d}(t), \\
u_{f f}(t)=\underbrace{\left[\mu_{1} \kappa_{2}(t)+1 \mu_{1} \kappa_{1}(t)+\mu_{2}\right]}_{C_{F F}(t)} x(t) \\
+\underbrace{\left[\mu_{1} \kappa_{3}(t)\right]}_{D_{F F}(t)} \ddot{y}_{d}(t),
\end{gathered}
$$

where $x(t)=\left[\begin{array}{cc}v(t) & \dot{v}(t)\end{array}\right]^{T}$, and $y_{d}(t)$ is the reference setpoint.

From the state-space model, it can be observed that all the matrices are time-dependent, hence resulting in the LTV feedforward controller. Note that the time-varying compliance function $C(t)$, and its derivatives $\dot{C}, \ddot{C}$ are included in the parameter values, see (11)-(13). Furthermore, the second time-derivative of $y_{d}$ is often known a priori, and as such available for calculation of the feedforward control signal $u_{f f}(t)$.

\section{FEASIBILITY CONDITIONS}

In this section, the dominant resonance frequency $\omega_{s}$ and compliance $C(t)$ will be coupled in order to maintain stability of the feedforward controller.
The feedforward controller should avoid any division by zero, i.e., $1+\omega_{s}^{2} m C(t) \neq 0$, see (11), (12) and (13). The resonance frequency $\omega_{s}$ ensures the feasibility of the feedforward controller under the following conditions:

$$
\begin{cases}\omega_{s}>0 & \text { if } C(t)>0 \\ 0<\omega_{s}<\frac{1}{\sqrt{-m C(t)}} & \text { if } C(t)<0 .\end{cases}
$$

In [13] additional conditions on $A_{F F}, B_{F F}, C_{F F}$ and $D_{F F}$ are presented ensuring stability of the LTV feedforward controller.

\section{NUMERICAL SIMULATION}

As an example of a position-dependent flexible structure, the damped Euler-Bernoulli beam is used for numerical investigation. This distributed parameter system is a step toward modeling the dynamics of a wafer stage system. The vertically moving Euler-Bernoulli beam is illustrated in Fig. 5. We denote the POI $r_{p}$, the length $L$, and the deflection of the beam at position $r_{p}$, or $y\left(t, r_{p}\right)$. The beam properties are mentioned in Table I. The partial differential equation

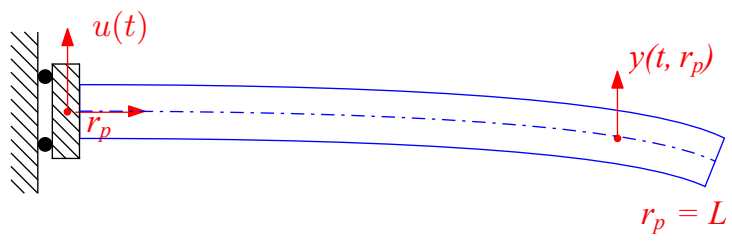

Fig. 5: The damped Euler-Bernoulli beam schematic

(PDE) describing the motion of the beam is given by

$$
\rho A \frac{\partial^{2} y\left(t, r_{p}\right)}{\partial t^{2}}+E I \frac{\partial^{4} y\left(t, r_{p}\right)}{\partial r_{p}^{4}}+c_{d} \frac{\partial^{5} y\left(t, r_{p}\right)}{\partial t \partial r_{p}^{4}}=0 .
$$

The four boundary conditions of the above PDE are the following:

$$
\begin{gathered}
\left.E I \frac{d^{3} y\left(t, r_{p}\right)}{d r^{3}}\right|_{r_{p}=0}=u(t), \\
\left.\frac{d y\left(t, r_{p}\right)}{d r}\right|_{r_{p}=0}=0, \\
\left.\frac{d^{2} y\left(t, r_{p}\right)}{d r^{2}}\right|_{r_{p}=L}=0, \\
\left.E I \frac{d^{3} y\left(t, r_{p}\right)}{d r^{3}}\right|_{r_{p}=L}=0 .
\end{gathered}
$$

This distributed parameter system contains one RB and an infinite number of NRB modes which translate into infinite resonances. The time domain transfer of the NRB dynamics is the summation of second order filters in association with each NRB resonance, multiplied by a time-varying compliance of each NRB mode. It is given by

$$
G\left(p, r_{p}\right)=\frac{1}{m p^{2}}+\sum_{i=1}^{\infty} \frac{c_{i}\left(r_{p}\right) \omega_{i}^{2}}{p^{2}+2 \omega_{i} \zeta_{i} p+\omega_{i}^{2}},
$$

where $\omega_{i}, \zeta_{i}$ and $c_{i}\left(r_{p}\right)$ are the resonance, damping coefficient and the compliance in association with each NRB 
mode, respectively. $c_{i}\left(r_{p}\right)$ is calculated via modal approximation which is a high-order approximation method for distributed parameter systems [10]. The derivation of $c_{i}\left(r_{p}\right)$ is skipped here due to lack of space [11].

In a practical case study of wafer stage problem, the

TABLE I: Euler-Bernoulli beam properties

\begin{tabular}{cc|}
\hline Property & Value \\
\hline Length & $L=0.6[\mathrm{~m}]$ \\
\hline Cross-sectional area & $A=h^{2}=10^{-4}\left[\mathrm{~m}^{2}\right]$ \\
\hline Mass density & $\rho=7.75 \times 10^{3}\left[\frac{\mathrm{kg}}{\mathrm{m}^{3}}\right]$ \\
\hline Young's modulus & $E=2 \times 10^{10}\left[\frac{\mathrm{kg}}{\mathrm{m} \cdot \mathrm{s}^{2}}\right]$ \\
\hline Second moment of inertia & $I=\frac{h^{4}}{12}=\frac{10^{-4}}{12}\left[\mathrm{~m}^{4}\right]$ \\
\hline Kelvin-Voigt damping & $c_{d}=0.4625$ \\
\hline Dominant damping coefficient & $\zeta_{1}=0.15$ \\
\hline
\end{tabular}

frequency domain mostly does not exceed $10 \mathrm{kHz}$, which only consists of the first resonance of the system. Actually, the full flexible dynamics appear only at high frequencies. Hence, for simulation purposes, the considered model for the Euler-Bernoulli beam is truncated to five NRB modes. The contribution of more NRB modes has been studied and was proved that more NRB modes do not affect the result of simulation since at low-working frequency domain, only the first resonance of NRB modes plays the major role. Thus, the model is given by

$$
G\left(p, r_{p}\right)=\frac{1}{m p^{2}}+\sum_{i=1}^{5} \frac{c_{i}\left(r_{p}\right) \omega_{i}^{2}}{p^{2}+2 \omega_{i} \zeta_{i} p+\omega_{i}^{2}} .
$$

The model-based feedforward control is based on the approximated plant at low frequencies and is given by

$$
\tilde{G}_{\omega}(p, t)=\frac{1}{m p^{2}}+C\left(r_{p}\right) \frac{\omega_{s}^{2}}{p^{2}+2 \omega_{s} \zeta_{s} p+\omega_{s}^{2}} .
$$

Due to the intended frequency interval, the dominant resonance frequency of the approximated system $\omega_{s}$ is considered to be the first resonance of the system $\omega_{s}=\omega_{1}$, and correspondingly, $\zeta_{s}$ is the damping coefficient of the first NRB mode $\zeta_{s}=\zeta_{1} . C\left(r_{p}\right)$ is the full compliance of the beam which compensates fully the NRB modes at low frequencies. It can be calculated by a zero limit calculation of the NRB parts of the transfer function. For this specific example, it has been calculated as being [11]:

$$
C\left(r_{p}\right)=10^{-6}\left(-\frac{10}{24} r_{p}^{4}+r_{p}^{3}-\frac{9}{10} r_{p}^{2}+\frac{81}{1250}\right) .
$$

(22) gives that the compliance function of the beam is dependent on the POI $r_{p}(t)$. In order to indicate the POI as a function of time, $r_{p}(t)$ also needs to be taken into account. In this example, $r_{p}(t)$ is chosen based on the conditions of (16).

$$
r_{p}(t)=0.1(1-\cos (12.5 \pi t)) .
$$

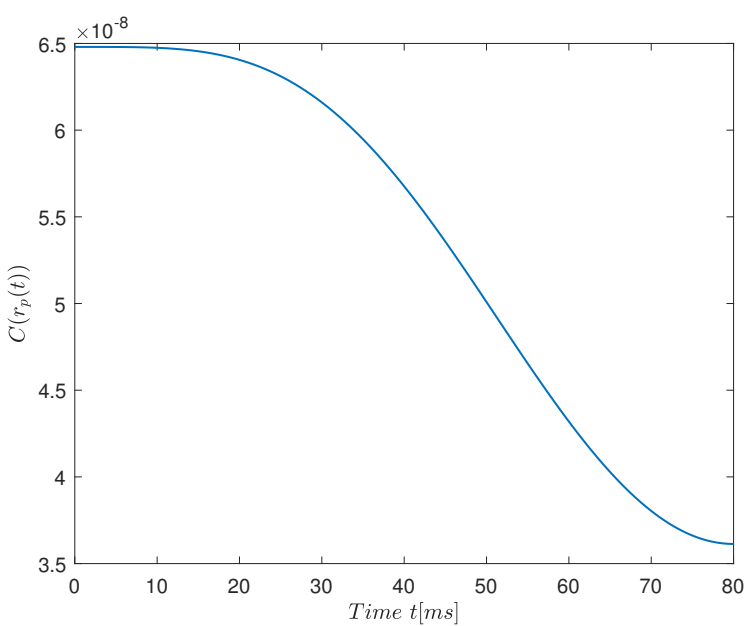

Fig. 6: Time-varying compliance function of the positiondependent flexible beam with specifications of Table I

The time-varying compliance function of the damped EulerBernoulli beam is plotted in Fig. 6. It shows the transversal deflection of the beam caused by two effects. Firstly, the beam is actuated by a constant force $u(t)$ in vertical direction. Secondly, it considers a sinusoidal wave function of the POI. Furthermore, the fourth-order tracking position $y_{d}$ shown in Fig. 7, is used as an input signal of the feedforward controller. The region specified by vertical dashed lines is the wafer scanning interval which occurs at constant velocity. In
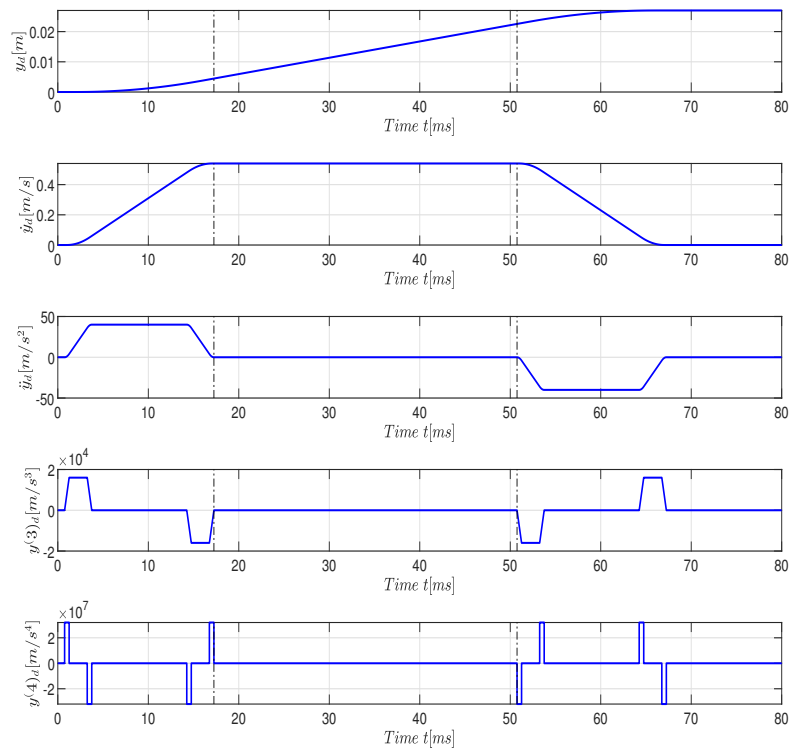

Fig. 7: Fourth-order reference setpoint $y_{d}$

order to correct the minor remaining error of the output, an LTI PID feedback controller is tuned via manual loopshaping [12].

Simulation results are illustrated in Fig. 8. As a good in- 
dicator of the control performance, the control signal $u_{f f}$ is checked for preserving the input signal of the reference setpoint $y_{d}$. Since, the proposed LTV feedforward control is an LTV extension of the snap feedforward control and both model-based feedforward controllers include the acceleration term, for a better observation of the feedforward controller output difference, Fig. 8(a) depicts the control effort signal $u_{f f}$ excluding the acceleration term $u_{a c c}$. As can be seen, the discontinuous, step-like control input of snap feedforward control leads to excitation of NRB modes, however the smooth signal produced by the LTV feedforward control induce much less excitation of NRB modes. Fig. 8(b) shows the tracking error of the model in closed loop using the LTV feedforward control and a traditional (LTI) acceleration-snap feedforward control. It illustrates that the LTV feedforward control potentially reduces the error in the scanning interval. The error reduction in the scanning interval is obtained by essentially providing feedforward controller output (unequal to zero) also in the interval of constant velocity, where the accelerations are zero.

\section{CONCLUSIONS}

A model-based feedforward control is obtained for a flexible structure with a time-varying POI. The controller which is an extension of snap feedforward control [1], considers both the zero frequency contribution as well as the dominant resonant dynamics of the position-dependent flexible structure. Simulation studies were carried out for a damped Euler-Bernoulli beam. Furthermore, the tracking error of the system with the LTV feedforward control results in a fairly small error in comparison with snap feedforward control due to the presence of the smooth feedforward control signal. The proposed LTV feedforward control targets at being developed irrespective to POI, which can be the scope of future works.

\section{REFERENCES}

[1] M. Boerlage, MIMO jerk derivative feedforward for motion systems, American Control Conf., Minneapolis, USA, pp. 3892-3897, June 2006.

[2] T. Oomen, R. van Herpen, S. Quist, M. van de Wal, O. Bosgra, and M. Steinbuch. Connecting System identification and robust control for next-generation motion control of a wafer stage, IEEE Trans. Control Syst. Technol., vol. 22, no. 1, 2014.

[3] P. Lambrechts, M. Boerlage, and M. Steinbuch, Trajectory planning and feedforward design for electromechanical motion systems, Control Eng. Pract., vol. 13, no. 2, pp. 145-157, 2005.

[4] H., Butler, Adaptive feedforward for a wafer stage in a lithographic tool, IEEE Trans. Control Syst. Technol., vol. 21, no. 3, pp. 875-881, 2013.

[5] M. Sato, Gain-scheduled inverse system and filtering system without derivatives of scheduling parameters, Proceedings of the 2003 American Control Conf., Denver, USA, pp. 4173-4178, June 2003.

[6] M. Sato, Inverse system design for LPV systems using parameter dependent Lyapunov functions, Automatica, vol. 44, no. 4, pp. 10721077, 2008.

[7] Y. Kasemsinsup, M. F. Heertjes, H. Butler, and S. Weiland, Exact plant inversion of flexible motion systems with a time-varying stateto-output map, European Control Conf., Aalborg, Denmark, pp. 24832488, June 2016.

[8] Y. Kasemsinsup, R. Romagnoli, M.F. Heertjes, S. Weiland, and H. Butler, Reference-tracking feedforward control design for linear dynamical systems through signal decomposition, American Control Conf., Seattle, Washington pp. 2387-2392, May 2017.

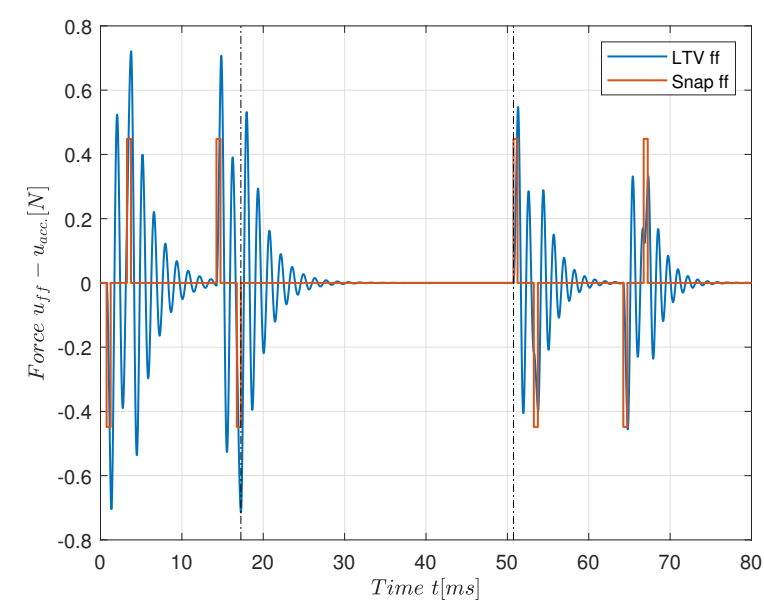

(a) Feedforward control signal $u_{f f}-u_{a c c}$.

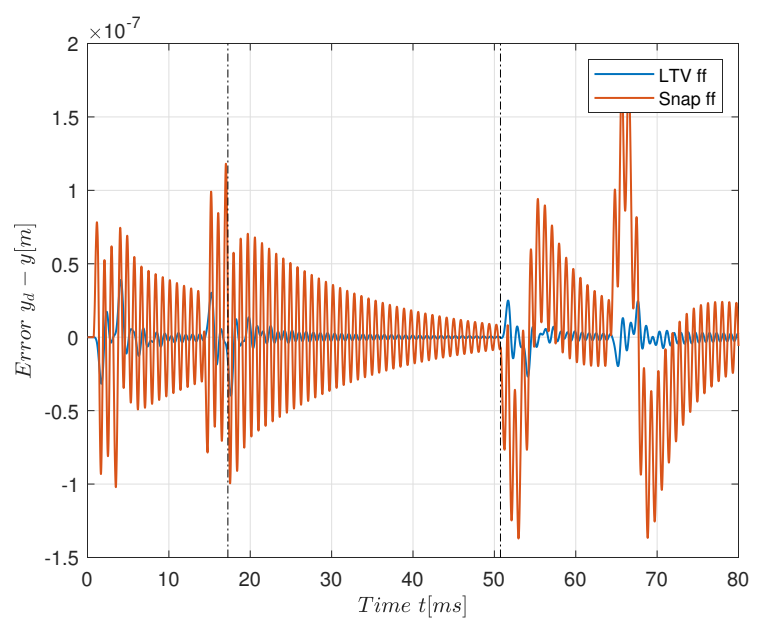

(b) Position tracking error of the system with the LTV feedforward control and snap feedforward control.

Fig. 8: Simulation result with the LTV feedforward control and snap feedforward control. The scanning interval is enclosed by the vertical dashed lines.

[9] Y. Kasemsinsup, A.F. Ardyanto, H. Butler, M.F. Heertjes, and S. Weiland, American Control Conf., Milwaukee, USA, pp. 6690-6696, June 2018.

[10] R. Curtain, and K. Morris. Transfer Functions of Distributed Parameter Systems: A Tutorial, Automatica, vol. 45, no. 5, pp. 1101-1116, 2009.

[11] N. Kontaras, M.F. Heertjes, and H. Zwart, Continuous compliance compensation of position dependent flexible structures. IFACPapersOnLine, vol. 49, no. 13, pp. 76-81, 2016.

[12] N. Kontaras, M.F. Heertjes, H. Zwart, and M. Steinbuch, A compliance feedforward scheme for a class of LTV motion systems. 2017 American Control Conf., Seattle, USA, pp. 4504-4509, May 2017.

[13] N. Kontaras, M.F. Heertjes, H. Zwart, and M. Steinbuch, Resonantdynamics LTV feedforward for flexible motion systems. 2018 American Control Conf., Milwaukee, USA, pp. 6012-6017, June 2018.

[14] T. Glad, L. Ljung, Control Theory: Multivariable and Nonlinear Methods, CRC Press, FL, USA, 2000. 